\title{
Fatores associados à incoagulabilidade sangüínea no envenenamento por serpentes do gênero Bothrops
}

\author{
Risk factors associated with coagulation abnormalities \\ in Bothrops envenoming
}

\author{
Ricardo Borges de Oliveira1, Lindioneza Adriano Ribeiro'e Miguel Tanús Jorge1
}

\begin{abstract}
Resumo Com o objetivo de conhecer fatores associados à incoagulabilidade sangüínea no envenenamento botrópico, foram obtidas informações de 2.991 prontuários médicos de pacientes atendidos no Instituto Butantan de 1981 a 1990. Associaram-se positivamente à incoagulabilidade sangüínea $(p<0,05)$ : picadas no final do ano e em extremidades dos membros inferiores; dor, edema e equimose local; hemorragia e choque; dose de antiveneno; tempo do acidente à chegada ao Instituto Butantan. Associaram-se negativamente à incoagulabilidade $(p<0,05)$ : tamanho de Bothrops jararaca; uso de torniquete; tempo entre a chegada ao Instituto Butantan e o início da administração do antiveneno. Não se associaram à incoagulabilidade ( $p>0,05)$ : horário do acidente; presença de presa recém-deglutida no tubo digestivo da serpente; sexo e idade do paciente; ocorrência de bolha, necrose, abscesso e incisão local, amputação, insuficiência renal e óbito. Pode-se concluir que, embora a incoagulabilidade sangüínea apresente associação com manifestações precoces do envenenamento, não tem boa associação com a evolução clínica do paciente.
\end{abstract}

Palavras-chaves: Bothrops jararaca. Coagulação sangüínea. Fatores prognósticos.

Abstract This study aimed at assessing, in the envenoming by Bothrops, factors that are associated with blood incoagulability. Information was obtained from the charts of 2,991 patients admitted to Instituto Butantan, from 1981 to 1990. Factors positively associated with blood incoagulability $(p<0.05)$ were: snake bite in the late months of the year; bites in the distal segments of the lower limbs; pain, edema, and bruising at the bite site; systemic bleeding and shock; dose of antivenom administered; time between bite and admission to Instituto Butantan. Size of the snake Bothrops jararaca; use of a tourniquet; and time between arrival to Instituto Butantan and start of the antivenom administration were negatively associated with blood incoagulability $(p<0,05)$. Factors not associated with blood incoagulability ( $p>0.05$ ) were: time of the bite; presence of recently swallowed prey in the snake gut; gender and age of the patient; blister, necrosis, and abscess at the bite site; occurrence of amputation, renal failure and death; presence of an incision at the bite site. We conclude that although blood incoagulability is associated with early manifestations of Bothrops envenoming, it is not associated with the clinical outcome.

Key-words: Bothrops jararaca. Blood coagulability. Prognostic factors.

No Brasil, aproximadamente $90 \%$ dos acidentes por serpentes peçonhentas notificados são causados por Bothrops 6 .

O veneno botrópico causa lesão nos tecidos da região anatômica picada ${ }^{2}$ por ação de diversos componentes, tais como, fosfolipases ${ }^{8} 10 \mathrm{e}$ metaloproteinases ${ }^{129} 4752$. Provoca inflamação 3035 e destruição ${ }^{811} 123536$ desses tecidos, sendo também observado dano na parede de pequenos vasos sangüíneos ${ }^{32} 3336$.

Na região anatômica picada ocorrem dor, edema, equimose, flictenas e necrose ${ }^{31344245}$. A destruição de tecidos e a inoculação de bactérias, juntamente com o veneno, podem levar à infecção e à formação de abscessos ${ }^{18} 2022$. Distúrbios funcionais permanentes e até mesmo a perda do membro acometido podem

\footnotetext{
1. Faculdade de Medicina da Universidade Federal de Uberlândia, Uberlândia, MG, Brasil.

Endereço para correspondência: Prof. Miguel Tanús Jorge. Dept ${ }^{\circ}$ de Clínica Médica/FM/UFU. Av. Pará 1720, Campus Umuarama, 38400 902, Uberlândia, MG, Brasil.

Tel: 34 3218-2224; Fax 34 3218-2349.

e-mail: miglind@ufu.br

Recebido para publicação em 22/7/2002

Aceito em 22/8/2003
} 


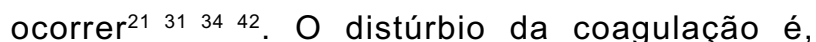
provavelmente, a manifestação sistêmica mais comum que, às vezes, exterioriza-se clinicamente por sangramentos distantes dos ferimentos causados pelos dentes inoculadores ${ }^{42}$. O óbito ocorre em casos de envenenamentos graves em pacientes que apresentam insuficiência renal, respiratória e choque ${ }^{37}$. A maioria, entretanto, evolui para a cura sem seqüelas ${ }^{31} 3442$.

Embora a alteração da coagulação ocorra precocemente, parece não apresentar boa associação com a destruição tecidual na região anatômica picada ${ }^{3840}$.
Isso levou à sua retirada dos critérios para a classificação quanto à gravidade do envenenamento, com o objetivo de se administrar dose adequada de soro 561951 . Por outro lado, em alguns estudos, foi observada associação entre distúrbio da coagulação e evolução para manifestações locais como necrose e abscesso ou mesmo para o óbito 3437434 .

O presente estudo teve como objetivo conhecer fatores epidemiológicos e clínicos que se associam à ocorrência de incoagulabilidade sangüínea no envenenamento por serpentes do gênero Bothrops.

\section{MATERIAL E MÉTODOS}

Foram obtidos dados clínicos e epidemiológicos de 2.991 prontuários médicos de pacientes picados por serpentes do gênero Bothrops, atendidos no Hospital Vital Brazil (HVB) do Instituto Butantan (IB), no período de 1981 a 1990. O HVB-IB atende somente a pacientes picados por animais peçonhentos, possuindo equipe médica especializada e prontuários dirigidos para a obtenção de informações sobre essas intoxicações exógenas.

O diagnóstico do acidente botrópico baseou-se na epidemiologia, nas manifestações clínicas e laboratoriais e, quando possível, na identificação da serpente por técnico habilitado do Laboratório de Herpetologia do IB. Algumas serpentes foram submetidas a necropsia para verificação do conteúdo do tubo digestivo a fim de avaliar a presença de presa recém-deglutida.

Os pacientes foram agrupados entre aqueles que permaneceram com o sangue coagulável (Grupo C) e aqueles que apresentaram incoagulabilidade sangüínea (Grupo I).
A avaliação da coagulação foi feita à admissão dos pacientes no HVB e repetida horas após, caso necessário. Era realizada por observação de alguns mililitros de sangue coletados em seringa plástica, colocados em dois tubos de vidro limpos e secos, em banho-maria a $37^{\circ} \mathrm{C}$ mas, às vezes, à temperatura ambiente. Enquanto um dos tubos era inclinado no sentido da posição horizontal, suave e periodicamente, com o objetivo de verificar se o sangue estava líquido e escorria por sua parede ou se havia coagulado, o outro tubo servia como controle do resultado obtido com o primeiro. Considerou-se como presença de sangue incoagulável a ausência de coagulação após a observação do sangue por 20 minutos $^{49}$.

A ocorrência de cada variável nos grupos C e I foi comparada pelo teste do qui-quadrado. A sazonalidade foi avaliada segundo a distribuição dos acidentes em cada bimestre. Quando necessário, foi utilizado o teste exato de Fisher. O nível de significância adotado foi de $5 \%$.

\section{RESULTADOS}

O mês de ocorrência do acidente mostrou associação com a incoagulabilidade sangüínea (Figura 1). Esta ocorreu em $48,7 \%, 44,3 \%, 48,2 \%$ e $47,2 \%$ dos pacientes picados, respectivamente, nos horários das 00:00h às 06:00, das 06:00h às 12:00, das 12:00h às 18:00 e das 18:00h às $24: 00$ ( $p>0,05$ ). Mediam mais do que 40 centímetros de comprimento $52,5 \%$ das serpentes que picaram os pacientes do grupo $\mathrm{C}$ e $36,3 \%$ das que picaram aqueles do grupo I $(p<0,05)$. Obteve-se informação sobre a presença de presas recém-deglutidas no tubo digestivo de 111 serpentes que picaram pacientes do grupo $C$ e de 94 daquelas que picaram os do grupo I. Havia presas em $9 \%$ das primeiras e em $9,6 \%$ das últimas $(p>0,05)$. Eram do sexo masculino $76,1 \%$ dos pacientes do grupo $C$ e $74,9 \%$ dos do grupo I ( $p>0,05)$. A incoagulabilidade sangüínea ocorreu em 50,5\%, 47,3\%, 45,5\%, 46,0\%, $44,6 \%, 45,9 \%$ e $47,5 \%$ dos pacientes, respectivamente, com 0 a 9, 10 a 19, 20 a 29, 30 a 39, 40 a 49, 50 a 59 e 60 ou mais anos de idade $(p>0,05)$. A incoagulabilidade sangüínea foi mais freqüente entre pacientes picados no pé ou tornozelo $(p<0,05)$ (Figura 2$)$. Dor, edema, equimose, bolha, necrose e abscesso ocorreram em, respectivamente, $92 \%, 91,8 \%, 48,8 \%, 12,3 \%, 14,6 \%$, $9,2 \%$ dos pacientes do grupo $\mathrm{C}$ e em $99,4 \%(p<0,05)$, $99,4 \%(p<0,05), 64,3 \%(p<0,05), 14,3 \%(p>0,05), 15,9 \%$ ( $p>0,05), 10,8 \%(p>0,05)$ daqueles do grupo I. Sangramento sistêmico, choque, insuficiência renal e óbito, ocorreram em, respectivamente, 5,3\%, 0,3\%,1,1\%, $0,2 \%$ dos pacientes do grupo $\mathrm{C}$ e em $19,8 \%(p<0,05)$, $1 \%(p<0,05), 1,5 \%(p>0,05), 0,1 \%(p>0,05)$ daqueles do grupo I. Evoluíram para amputação do membro acometido $0,4 \%$ dos pacientes do grupo $\mathrm{C}$ e $0,8 \%$ daqueles do grupo I $(p>0,05)$. Houve uso de torniquete em $41,4 \%$ dos pacientes do grupo $\mathrm{C}$ e em $36,7 \%$ daqueles do grupo I $(p<0,05)$. A realização de incisão local ocorreu em 3,9\% dos pacientes do grupo $\mathrm{C}$ e em $3,4 \%$ daqueles do grupo I $(p>0,05)$. Incoagulabilidade sangüínea foi menos comumente detectada nos pacientes atendidos muito precocemente ou muito tardiamente (Figura 3 ). Receberam soro dentro de uma hora após o atendimento no HVB, $71,7 \%$ dos pacientes do grupo C e $83,1 \%$ daqueles do grupo I $(p<0,05)$. Os pacientes do grupo $C$ receberam também doses menores de soro do que aqueles do grupo I (Figura 4). 


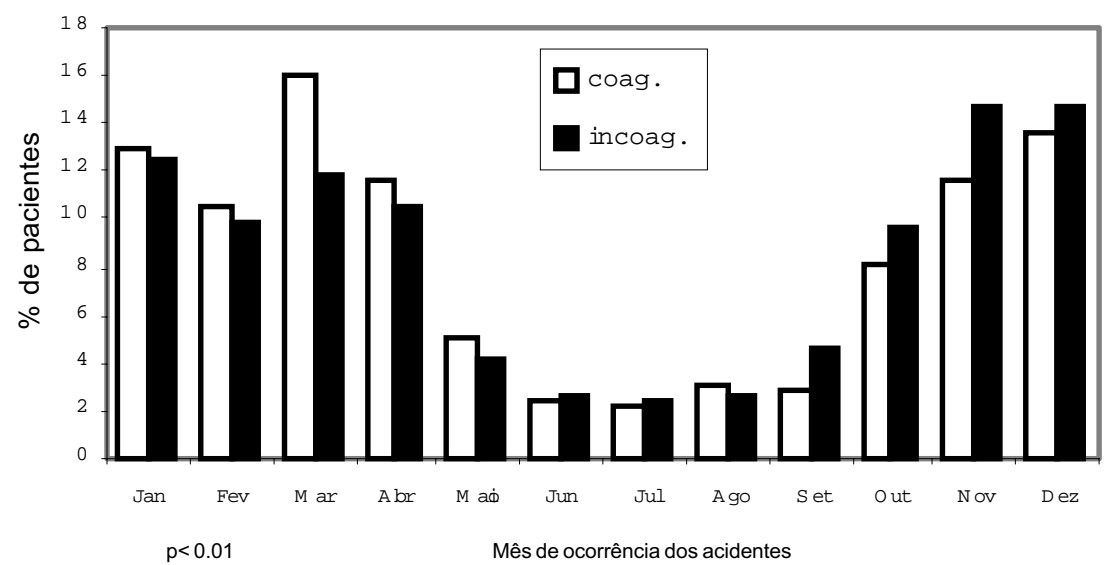

Figura 1 - Pacientes picados por Bothrops segundo o mês em que ocorreu o acidente e presença de incoagulabilidade sangüinea, HVB, 1981 a 1990.

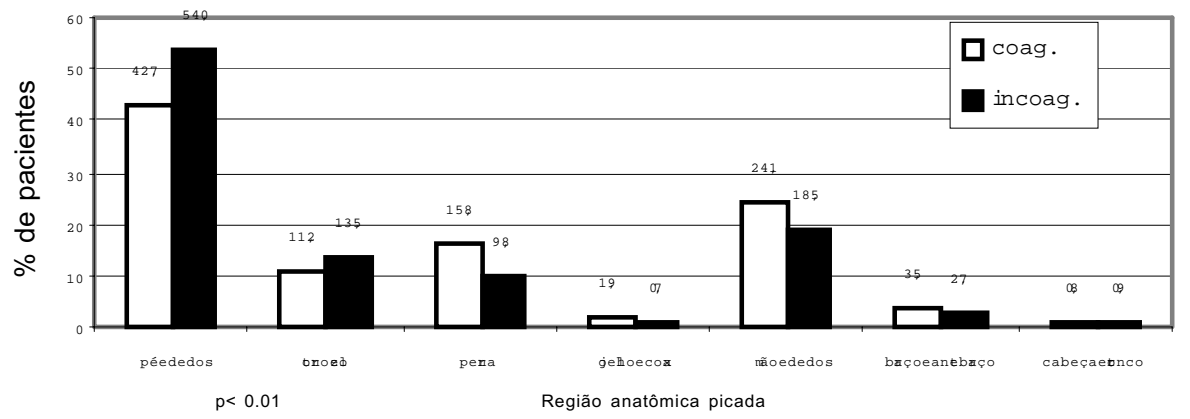

Figura 2 - Pacientes picados por Bothrops segundo região anatômica picada e ocorrência de incoagulabilidade sangüínea, HVB, 1981 a 1990.

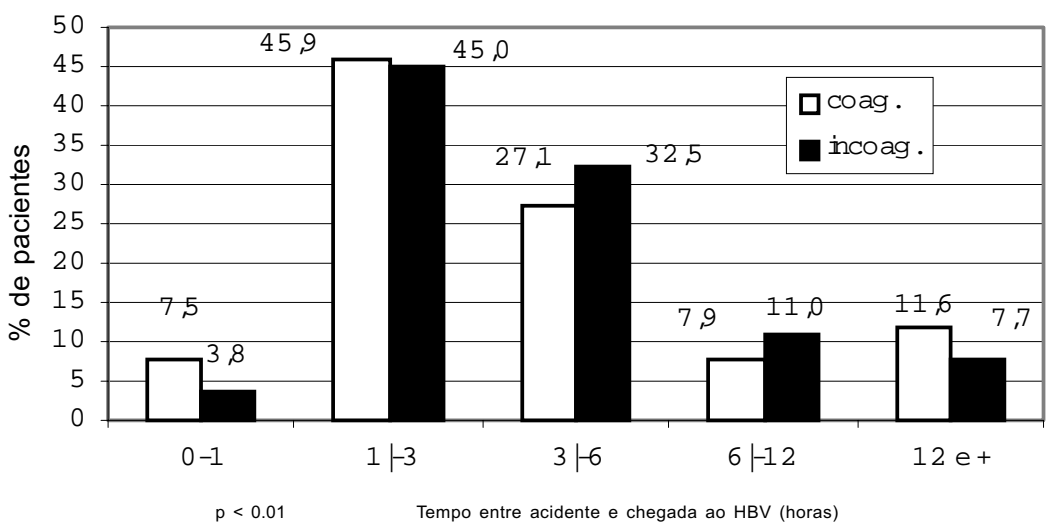

Figura 3 - Pacientes picados por Bothrops segundo o tempo entre acidente e chegada ao HVB e ocorrência incoagulabilidade sangüínea, HVB, 1981 a 1990. 


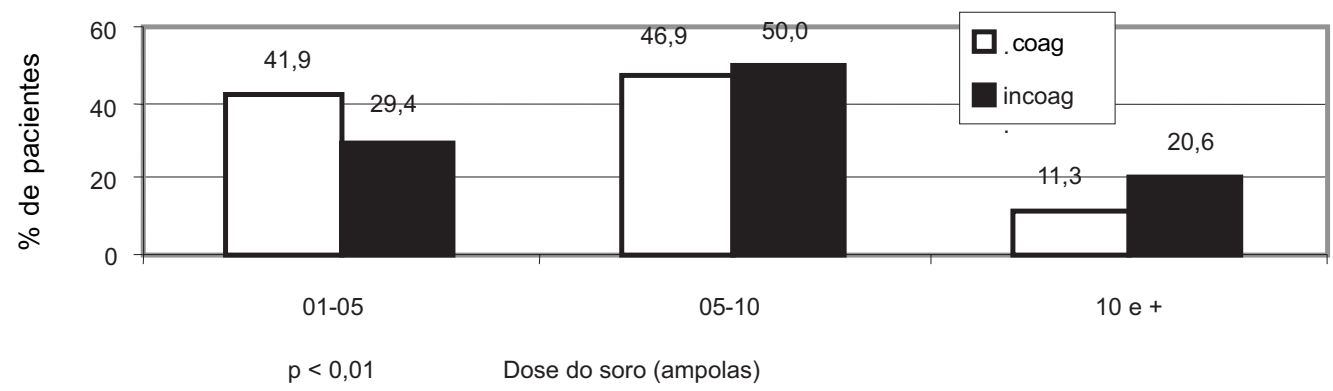

Figura 4 - Pacientes picados por Bothrops segundo dose do soro poliespecífico administrada e ocorrência de incoagulabilidade sangüínea, HVB, 1981 a 1990.

\section{DISCUSSÃO}

Serpentes do gênero Bothrops de pequeno porte, em relação àquelas de grande porte, costumam possuir veneno com maior atividade coagulante ${ }^{7346}$ por ativar mais intensamente os fatores II e $\mathrm{X}^{723}$. Conforme dados do presente estudo e de estudos anteriores ${ }^{28} 313941$, a ocorrência de incoagulabilidade sangüínea é também mais comum no envenenamento causado pelas serpentes de pequeno porte. Estas, quando da espécie B. jararaca, causam acidentes, que, proporcionalmente às de grande porte, ocorrem mais no final do que no início do $\mathrm{ano}^{41}$. Isso pode explicar a associação positiva entre acidentes nos últimos meses do ano e incoagulabilidade sangüínea. A ausência de associação entre o horário do acidente e a incoagulabilidade sanguínea é coerente com a falta de dados biológicos que sugiram essa possibilidade.

Já foi considerado que quando uma serpente pica após ter-se alimentado ou picado outra vítima, inocula menos veneno ${ }^{4}$ e em picadas por Crotalus viridis observou-se o contrário ${ }^{14}$. No presente estudo, em que pese o pequeno número de casos avaliados, não se observou associação positiva ou negativa entre alteração da coagulação e a presença de presa recém deglutida no tubo digestivo das serpentes.

O volume sangüíneo e o peso corporal variam com o sexo e a idade, sendo maior nos homens e menor nas mulheres e crianças ${ }^{13}$. Assim, haveria maior quantidade de veneno inoculado, proporcionalmente ao volume sangüíneo, no caso de mulheres e, sobretudo, crianças, em relação aos homens. Como, no presente estudo, não se observou associação entre sexo ou idade dos pacientes e incoagulabilidade sangüínea, pode-se pensar, conforme observado para Crotalus viridis ${ }^{15}$, na possibilidade das serpentes regularem a quantidade de veneno a ser inoculada de acordo com o tamanho do animal agredido.

Espécimes de Bothrops de grande porte picam porções mais altas dos membros inferiores com maior freqüência do que as menores e causam alterações da coagulação em suas vítimas menos freqüentemente 28313941 . Isso pode ser a explicação para a maior ocorrência de incoagulabilidade sangüínea em pacientes picados nas extremidades dos membros inferiores em relação àqueles picados acima do tornozelo.

Poucos pacientes que procuram assistência médica devido a picada apresentam-se sem dor e edema e, dentre esses, em muitos, talvez sequer tenha havido inoculação da peçonha. Pacientes sem dor e edema talvez não tenham apresentado alteração da coagulação porque, na realidade, não foram envenenados. Isso dificulta a interpretação da associação positiva de dor e edema com incoagulabilidade sangüínea.

Embora os sangramentos locais e até sistêmicos, decorrentes do envenenamento botrópico, tenham sido relacionados à ação das hemorraginas ${ }^{16} 242526274753$, é possível que a associação positiva encontrada de incoagulabilidade sangüínea com equimose e sangramento sistêmico tenha explicação no fato da primeira manifestação favorecer a ocorrência das demais.

Foi encontrada associação significativa entre atividades coagulante e necrotizante, ao se comparar venenos de várias espécies de Bothrops ${ }^{2}$, entretanto, essa associação não foi pesquisada para serpentes jovens em relação às adultas e já é conhecido que serpentes pequenas causam, em suas vítimas, maior freqüência de distúrbios da coagulação, enquanto serpentes grandes, maior freqüência de lesão tecidual local28 313941 . Justificase, assim, a ausência de associação entre bolha e necrose com incoagulabilidade sangüínea, observada no presente estudo, o que corrobora resultados de estudo realizado no Triângulo Mineiro ${ }^{34}$. Por meio de análise multivariada, entretanto, associação independente, embora fraca, foi observada ${ }^{43}$.

A ausência de associação da incoagulabilidade sangüínea com abscesso, verificada no presente estudo, tem a mesma explicação da não associação com necrose. A necrose dos tecidos favorece o aparecimento de infecções, com formação de abscessos 192022 . Como a amputação é resultado justamente da ocorrência de necrose e/ou infecção, da mesma forma, não se observou associação da sua 
ocorrência com a incoagulabilidade sangüínea, o que corrobora dados anteriores ${ }^{21}$. Vale ressaltar, entretanto, que um outro estudo mostrou ocorrência de associação de incoagulabilidade com abscesso ${ }^{34}$.

A associação positiva da incoagulabilidade sangüínea com a ocorrência de choque, encontrada no presente estudo, a não ser pelo maior volume de veneno inoculado e conseqüente maior gravidade do envenenamento em todos os seus aspectos, não tem explicação clara. A ausência de associação com insuficiência renal, por sua vez, corrobora a idéia de que a coagulopatia, devida a envenenamento por serpentes, costuma levar a exuberantes alterações laboratoriais e poucas repercussões clínicas ${ }^{54}$.

A existência de associação entre óbito e incoagulabilidade sangüínea ${ }^{37}$ não foi confirmada pelo presente estudo, mas o número de óbitos estudados não foi suficiente para essa avaliação.

Embora alguns autores considerem que não haja dados convincentes de que o torniquete possa retardar a absorção do veneno ${ }^{53}$, se isso for possível, a demora que acarretaria na difusão do veneno para os tecidos a jusante e para a corrente sangüínea, entre os pacientes em uso de torniquete poderia propiciar que fossem tratados com a soroterapia específica e ter o veneno neutralizado antes mesmo do sangue tornarse incoagulável. Isso explicaria o fato daqueles que usaram torniquete terem apresentado menor freqüência de incoagulabilidade sangüínea. Esses resultados, não autorizam o incentivo ao uso do torniquete pois, no envenenamento botrópico, esse procedimento está associado à ocorrência de necrose e abscesso na região anatômica picada ${ }^{40} 43$.

A associação entre incoagulabilidade sangüínea e uso de maiores doses de soro deve-se a que, no período em que os pacientes do presente estudo foram atendidos, recomendava-se maior dose de soro nos pacientes com sangue incoagulável ${ }^{50}$. Posteriormente, em um estudo realizado em área de predomínio de $B$. jararaca, não foi encontrada maior proporção de sangue incoagulável nos pacientes que apresentaram má evolução do envenenamento na região da picada ${ }^{38}$. A incoagulabilidade sangüínea foi, então, retirada dos critérios de classificação quanto à gravidade desses acidentes, com vistas a se determinar a dose de soro a ser administrada ${ }^{51}$. Além disso, doses de soro antibotrópico poliespecífico, significativamente menores do que as que vinham sendo preconizadas e usadas no HVB, mostraram-se suficientes para o tratamento dos distúrbios da coagulação de pacientes com envenenamento botrópico leve ou moderado em área de predominância de $B$. jararaca ${ }^{17}$. Ausência de associação entre incoagulabilidade sangüínea e gravidade do envenenamento na região anatômica picada pode ser explicada pelo fato do envenenamento por serpentes de menor comprimento estar mais freqüentemente associado a alterações da coagulação e aquele por serpentes de maior comprimento com danos teciduais locais ${ }^{41}$.

Segundo Rosenfeld, a difusão do veneno botrópico para a circulação sangüínea e a coagulopatia resultante da ação de seus componentes são geralmente graduais, de tal forma que, em muitos acidentes, a incoagulabilidade surge dentro de uma ou mais horas após a picada ${ }^{44} 45$. Assim, nas primeiras horas após o acidente, quanto maior o intervalo de tempo decorrido entre a picada e o atendimento, maior a possibilidade dos pacientes terem chegado ao hospital já com o sangue incoagulável, o que talvez possa justificar a associação positiva existente entre esse intervalo de tempo e a presença de incoagulabilidade sangüínea. Diferentemente, a existência de associação entre incoagulabilidade sangüínea e precocidade na administração do soro poliespecífico, após o atendimento, provavelmente se deve à preocupação do médico com a gravidade do envenenamento ou com um sangramento que tenha advindo.

Conclui-se que, no envenenamento por serpentes do gênero Bothrops, a incoagulabilidade sangüínea é mais comum quando o acidente ocorre nos últimos meses do ano, quando as picadas ocorrem nos segmentos distais dos membros inferiores e quando a serpente, se é $B$. jararaca, é de pequeno porte. Associa-se também a manifestações precoces do envenenamento botrópico, tais como, ocorrência de dor, edema, equimose, sangramento sistêmico e ao choque. Entretanto, não se associa à evolução clínica, no que se refere à ocorrência de bolha, necrose, abscesso, amputação, insuficiência renal e óbito, não se prestando, portanto, como bom indicador da evolução tardia do envenenamento. Pacientes que fazem uso de torniquete e que procuram tratamento mais precocemente desenvolvem incoagulabilidade sangüínea com menor freqüência.

\section{AGRADECIMENTOS}

Ao CNPq, pelo apoio financeiro, e à equipe de médicos e funcionários do Hospital Vital Brazil, pela assistência prestada aos pacientes.

\section{REFERÊNCIAS BIBLIOGRÁFICAS}

1. Aragon-Ortiz F, Gubensek F. Characterization of a metalloproteinase from Bothrops asper (terciopelo) snake venom. Toxicon 25:759-766, 1987.

2. Ferreira ML, Silva AMM, França FOS, Cardoso JL, Mota I. Toxic activities of venoms from nine Bothrops species and their correlation with lethality and necrosis. Toxicon 30:1603-1608, 1992.

3. Flores CA, Zappellini A, Prado-Franceschi J. Lipoxygenasederived mediators may be involved in in vivo neutrophil migration induced by Bothrops erythromelas and Bothrops alternatus venoms. Toxicon 31:1551-1559, 1993. 
4. Fonseca F. Animais peçonhentos. Empresa Gráfica da "Revista dos Tribunais", São Paulo,1949.

5. Fundação Nacional de Saúde. Manual de diagnóstico e tratamento de acidentes ofídicos. Ministério da Saúde, Brasília, 1987.

6. Fundação Nacional de Saúde. Manual de diagnóstico e tratamento de acidentes por animais peçonhentos. Ministério da Saúde, Brasília, 1998.

7. Furtado MFD, Maruyama M, Kamiguti AS, Antônio LC. Comparative study of nine Bothrops snake venoms from adult female snakes and their offspring. Toxicon 29:219-226, 1991.

8. Gutiérrez JM, Arce V, Brenes F, Chaves F. Changes in myofibrillar components after skeletal muscle necrosis induced by a myotoxin isolated from the venom of the snake Bothrops asper. Experimental and Molecular Pathology 52:25-36, 1990.

9. Gutiérrez JM, Chaves F. Efectos proteolitico, hemorragico y mionecrotico de los venenos de serpientes costarricenses de los generos Bothrops, Crotalus y Lachesis. Toxicon 18:315321, 1980.

10. Gutiérrez JM, Lomonte B, Chaves F, Moreno E, Cerdas L. Pharmacological activities of a toxic phospholipase $A$ isolated from the venom of the snake Bothrops asper. Comparative Biochemistry and Physiology 84C:159-164, 1986.

11. Gutiérrez JM, Ownby CL, Odell GV. Pathogenesis of myonecrosis induced by crude venom and a myotoxin of Bothrops asper. Experimental and Molecular Pathology 40:367379, 1984.

12. Gutiérrez JM, Romero M, Núñes J, Chaves F, Borkow G, Ovadia M. Skeletal muscle necrosis and regeneration after injection of $\mathrm{BaH} 1$, a hemorrhagic metalloproteinase isolated from the venom of the snake Bothrops asper (Terciopelo). Experimental and Molecular Pathology 62:28-41, 1995.

13. Guyton AC, Hall JE. Os compartimentos líquidos do corpo: líquído extracelular e intracelular; líquido intersticial e edema. In: Guyton AC, Hall JE (eds) Tratado de fisiologia médica. 9a edição. Guanabara Koogan, Rio de Janeiro, p. 277-290, 1997.

14. Hayes WK. Effects of hunger on striking, prey-handling, and venom expenditure of prairie rattlesnakes (Crotalus v. viridis). Herpetologica 49:305-310, 1993.

15. Hayes WK, Lavín-Murcio P, Kardong KV. Northern pacific rattlesnakes (Crotalus $v$. oreganus) meter venom when feeding on prey of different sizes. Copeia 2:337-343, 1995.

16. Hutton RA, Warrell DA. Action of snake venom components on the haemostatic system. Blood Reviews 7:176-189, 1993.

17. Jorge MT, Cardoso JLC, Castro SCB, Ribeiro LA, França FOS, Almeida MES, Kamiguti AS, Sano-Martins IS, Santoro ML, Mancau JEC, Warrell DA, Theakston RDG. A randomized 'blinded' comparison of two doses of antivenom in the treatment of Bothrops envenoming in São Paulo, Brazil. Transactions of The Royal Society of Tropical Medicine and Hygiene 89:111114, 1995.

18. Jorge MT, Mendonça JS, Ribeiro LA, Silva MLR, Kusano EJU, Cordeiro CLS. Flora bacteriana da cavidade oral, presas e veneno de Bothrops jararaca: possível fonte de infecção no local da picada. Revista do Instituto de Medicina Tropical de São Paulo 32:6-10, 1990.
19. Jorge MT, Ribeiro LA. Acidentes por serpentes peçonhentas do Brasil. Revista da Associação Médica Brasileira 36:66-77, 1990.

20. Jorge MT, Ribeiro LA. Infections in the bite site after envenoming by snakes of the Bothrops genus. Journal of Venomous Animals and Toxins 3:264-272, 1997

21. Jorge MT, Ribeiro LA, O'connell JL. Prognostic factors for amputation in the case of envenoming by snakes of the Bothrops genus (Viperidae). Annals of Tropical Medicine and Parasitology 93:401-408, 1999.

22. Jorge MT, Ribeiro LA, Silva MLR, Kusano EJU, Mendonça JS. Microbiological studies of abscesses complicating Bothrops snakebite in humans: a prospective study. Toxicon 32:743748, 1994.

23. Kamiguti AS. Atividades coagulante, inflamatória e proteolítica dos venenos de Bothrops Jararaca recém-nascida e adulta. Tese de Mestrado, Universidade de São Paulo, São Paulo, SP, 1988.

24. Kamiguti AS, Cardoso JLC, Theakston RDG, Sano-Martins IS, Hutton RA, Rugman FP, Warrell DA, Hay CRM. Coagulopathy and haemorrhage in human victims of Bothrops jararaca envenoming in Brazil. Toxicon 29:961-972, 1991.

25. Kamiguti AS, Hay CRM, Theakston RDG, Zuzel M. Insights into the mechanism of haemorrhage caused by snake venom metalloproteinases. Toxicon 34:627-642, 1996.

26. Kamiguti AS, Rugman FP, Theakston RDG, França FOS, Ishii $\mathrm{H}$, Hay CRM. The role of venom haemorrhagin in spontaneous bleeding in Bothrops jararaca envenoming. Thrombosis and Haemostasis 67:484-488, 1992.

27. Kamiguti AS, Theakston RDG, Desmond H, Hutton RA. Systemic haemorrhage in rats induced by a haemorrhagic fraction from Bothrops jararaca venom. Toxicon 29:1097-1105, 1991.

28. Kouyoumdjian JA, Polizelli C. Acidentes ofídicos causados por Bothrops moojeni: correlação do quadro clínico com o tamanho da serpente. Revista do Instituto de Medicina Tropical de São Paulo 31:84-90, 1989.

29. Lomonte B, Gutiérrez JM, Borkow G, Ovadia M, Tarkowski A Hanson LA. Activity of hemorrhagic metalloproteinase $\mathrm{BaH}-1$ and myotoxin II from Bothrops asper snake venom on capillary endothelial cells in vitro. Toxicon 32:505-510, 1994.

30. Lomonte B, Tarkowski A, Hanson LA. Host response to Bothrops asper snake venom. Analysis of edema formation, inflammatory cells, and cytokine release in a mouse model. Inflammation 17:93-105,1993.

31. Milani-Júnior R, Jorge MT, Ferraz-de-Campos FP, Martins FP, Bousso A, Cardoso JLC, Ribeiro LA, Fan HW, França FOS, Sano-Martins IS, Cardoso D, Fernandez ICOF, Fernandes JC Aldred VL, Sandoval MP, Puorto G, Theakston RDG, Warrell DA. Snake bites by the jararacuçu (Bothrops jararacussu): clinicopathological studies of 29 proven cases in São Paulo State, Brazil. Quarterly Journal of Medicine 90:323-334, 1997.

32. Moreira L, Borkow G, Ovadia M, Gutiérrez JM. Pathological changes induced by $\mathrm{BaH} 1$, a hemorrhagic proteinase isolated from Bothrops asper (terciopelo) snake venom, on mouse capillary blood vessels. Toxicon 32:976-987, 1994.

33. Moreira L, Gutiérrez JM, Borkow G, Ovadia M. Ultrastructural alterations in mouse capillary blood vessels after experimental 
injection of venom from the snake Bothrops asper (terciopelo). Experimental and Molecular Pathology 57:124-133, 1992.

34. Nishioka SA, Silveira PVP. A clinical and epidemiologic study of 292 cases of lance-headed viper bite in a Brazilian teaching hospital. The American Journal of Tropical Medicine and Hygiene 47:805-810, 1992.

35. Pérez OA, Koscinczuk P, Negrette MS, Teibler P, Ruíz R. Effects of Bothrops alternatus venom of Argentina on muscle and different organs in mice. Acta Physiologica Pharmacologica et Therapeutica Latinoamericana 46:97-102, 1996.

36. Queiroz LS, Santo Neto H, Assakura MT, Reichl AP, Mandelbaum FR. Muscular lesions induced by a hemorrhagic factor from the Bothrops neuwied snake venom. Brazilian Journal of Medical and Biological Research 18:337-340, 1985.

37. Ribeiro LA, Albuquerque MJ, Pires-de-Campos VAF, Katz G, Takaoka NY, Lebrão ML, Jorge MT. Óbitos por serpentes peçonhentas no Estado de São Paulo: avaliação de 43 casos, 1988/93. Revista da Associação Médica Brasileira 44:312-318, 1998.

38. Ribeiro LA, Jorge MT. Fatores prognósticos em acidentes por serpentes do gênero Bothrops. In: Resumos do XXI Congresso da Sociedade Brasileira de Medicina Tropical, São Paulo p. 28, 1985.

39. Ribeiro LA, Jorge MT. Alteração do tempo de coagulação sangüínea em pacientes picados por serpente Bothrops jararaca adulta e filhote. Revista do Hospital de Clínicas; Faculdade de Medicina da Universidade de São Paulo 44:143145, 1989.

40. Ribeiro LA, Jorge MT. Fatores prognósticos da evolução das manifestações locais em acidentes por serpentes do gênero Bothrops. Revista da Sociedade Brasileira de Medicina Tropical 22:68-69, 1989.

41. Ribeiro LA, Jorge MT. Epidemiologia e quadro clínico dos acidentes por serpentes Bothrops jararaca adultas e filhotes. Revista do Instituto de Medicina Tropical de São Paulo 32:436442, 1990.

42. Ribeiro LA, Jorge MT. Acidente por serpentes do gênero Bothrops: série de 3.139 casos. Revista da Sociedade Brasileira de Medicina Tropical 30:475-480, 1997.

43. Ribeiro LA, Jorge MT, Lebrão ML. Prognostic factors for local necrosis in Bothrops jararaca (Brazilian pit viper) bites. Transactions of The Royal Society of Tropical Medicine and Hygiene 95:630-634, 2001.
44. Rosenfeld G. Moléstias por venenos animais. Pinheiros Terapeutico 17:3-15, 1965.

45. Rosenfeld G. Symptomatology, pathology, and treatment of snake bites in South America. In: Bucherl W, Buckley EE (eds) Venomous animals and their venoms. Academic Press, New York, p. 345-384, 1971.

46. Rosenfeld G, Hampe OG, Kelen EMA. Coagulant and fibrinolytic activity of animal venoms; determination of coagulant and fibrinolytic index of different species. Memórias do Instituto Butantan 29:143-163, 1959.

47. Rucavado A, Lomonte B, Ovadia M, Gutiérrez JM. Local tissue damage induced by BaP1, a metalloproteinase isolated from Bothrops asper (terciopelo) snake venom. Experimental and Molecular Pathology 63:186-199, 1995.

48. Saborío P, González M, Cambronero M. Accidente ofídico en niños en Costa Rica: epidemiología y detección de factores de riesgo en el desarrollo de absceso y necrosis. Toxicon 36:359-366, 1998.

49. Sano-Martins IS, Fan HW, Castro SCB, Tomy SC, França FOS, Jorge MT, Kamiguti AS, Warrell DA, Theakston RDG. Reliability of the simple 20 minute whole blood clotting test (WBCT 20) as an indicator of low plasma fibrinogen concentration in patients envenomed by Bothrops snakes. Toxicon 32:1045-1050, 1994.

50. Secretaria da Saúde do Estado de São Paulo. Manual para atendimento dos acidentes humanos por animais peçonhentos. Coordenadoria de Serviços Técnicos Especializados. Instituto Butantan. Hospital Vital Brasil. São Paulo, 1982.

51. Secretaria da Saúde do Estado de São Paulo. Manual de vigilância epidemiológica; acidentes por animais peçonhentos; identificação, diagnóstico e tratamento. Centro de Vigilância Epidemiológica "Professor Alexandre Vranjac". Instituto Butantan. São Paulo, 1993

52. Serrano SM, Sampaio CA, Mandelbaum FR. Basic proteinases from Bothrops moojeni (caissaca) venom ${ }^{-}$II. Isolation of the metalloproteinase MPB. Comparison of the proteolytic activity on natural substrates by MPB, MSP 1 and MSP 2. Toxicon 31:483-492, 1993

53. Theakston RDG, Kamiguti AS. Viper envenoming evaluation of treatment by restoration of haemostasis and venom clearance. Journal of venomous animals and Toxins 4:94-111, 1998.

54. Williams EC, Mosher DF. Disseminated intravascular coagulation. In: Hoffman R, Benz Jr EJ, Shattil SJ, Furie B, Cohen HJ, Silberstein LE (eds) Hematology, basic principles and practice. $2^{\text {nd }}$ edition. Churchill Livingstone, New York, p. 1758-1769, 1995. 